\title{
Case Report: Cytarabine-Induced Pericarditis and Pericardial Effusion
}

\author{
Rino Sato, MD and Robert Park, MD
}

\section{INTRODUCTION}

Cytarabine (cytosine arabinoside, Ara-C) is an antimetabolite analogue of cytidine that is used as a chemotherapeutic agent for the treatment of acute myelogenous leukemia and lymphocytic leukemias ${ }^{1}$. The most common side effects of this therapy include myelosuppression, pancytopenia, hepatotoxicity, gastrointestinal ulceration with bleeding, and pulmonary infiltrates ${ }^{2}$ Cardio-pulmonary complications of cytarabine therapy are uncommon, but include supraventricular and ventricular arrhythmias, sinus bradycardia, and recurrent heart failure ${ }^{2,3}$. Occasionally, patients may develop pericarditis leading to pericardial tamponade, which can be fatal. We report a case of cytarabine-induced pericarditis and pericardial effusion to increase awareness about this serious side effect of cytarabine and review the current literature.

\section{CASE PRESENTATION}

A 44-year-old male with hypertension, obstructive sleep apnea, stage 3 chronic kidney disease, and recent admissions for symptomatic anemia of unknown etiology presented to the hospital after his outpatient lab work revealed a leukocytosis of 75,000 cells/ $\mu \mathrm{l}$, thrombocytopenia of 117,000 platelets/ $\mu$, and anemia with hemoglobin of $6.4 \mathrm{~g} / \mathrm{dl}$. An inpatient bone marrow biopsy revealed acute myeloid leukemia. A baseline transthoracic echocardiogram was obtained in preparation for inpatient chemotherapy, and demonstrated mild global left ventricular dysfunction with ejection fraction of $40 \%$. The cardiomyopathy was attributed to his underlying hypertension or sleep apnea, and not coronary artery disease based on a normal coronary computed tomography (CT) angiogram. The patient was started on induction therapy with high-dose cytarabine therapy at $3 \mathrm{~g} / \mathrm{m}^{2}$ every twelve hours without an anthracycline agent such as doxorubicin.

On day 5 of cytarabine therapy, the patient developed non-radiating sharp chest pain that worsened with inspiration and palpation. He had no cough or sputum production. His cardiac exam revealed a tri-phasic, high-pitched friction rub best heard over the left lower sternal border. He was normotensive, did not have pulsus paradoxus, and had minimally distended jugular veins. An electrocardiogram revealed widespread concave ST-elevation and PR-depression in the limb leads (I, II, III, avF) and precordial leads (V5-V6) concerning for acute pericarditis (Figure 1). The patient also had an elevated troponin T to $0.07 \mathrm{ng} / \mathrm{ml}$ (normal <0.01) and an elevated pro-BNP of $2080 \mathrm{pg} / \mathrm{ml}$ (normal <125) in the setting of acute on chronic kidney disease. A limited transthoracic echocardiogram revealed a small-to-moderate pericardial effusion along the inferolateral and inferior aspects of the left ventricle (Figure 2). There was no evidence of pleuritis on chest $x$-ray or chest CT. The patient was transferred to the cardiac intensive care unit for further management.

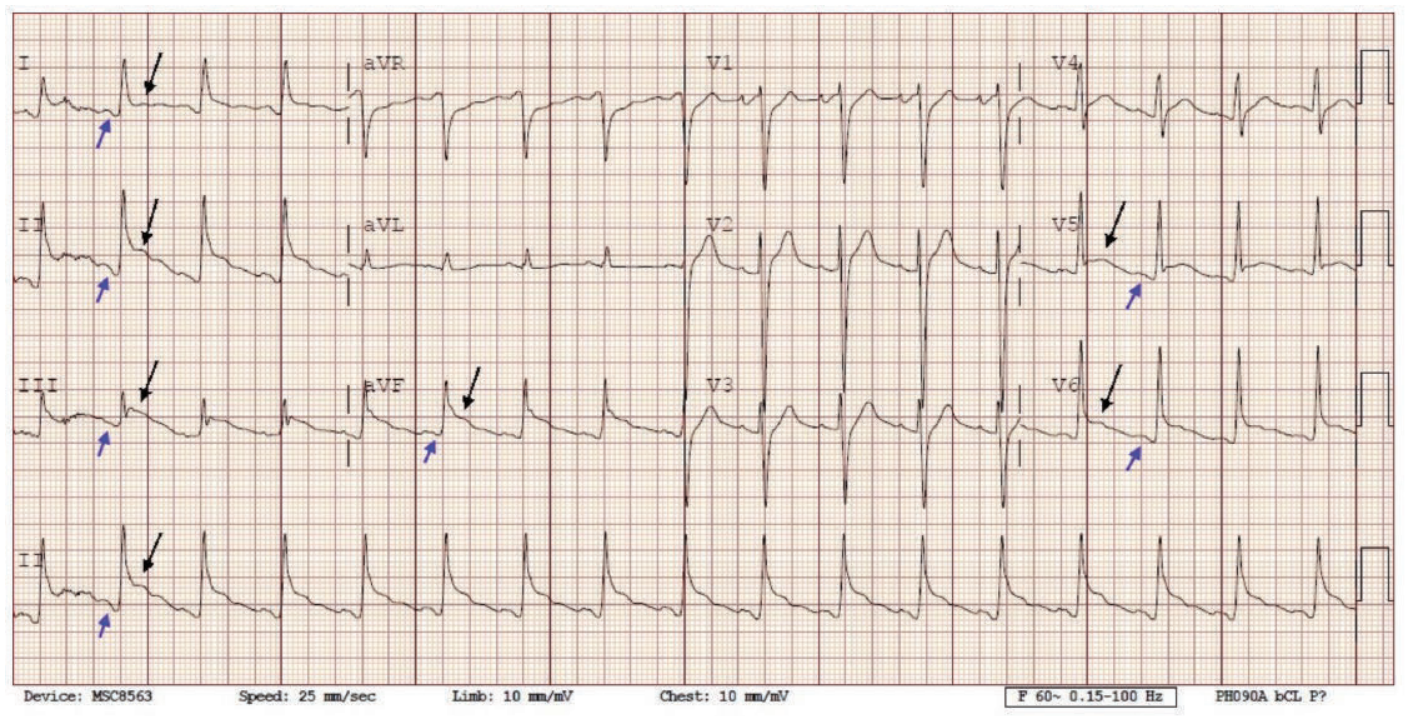

Figure 1. A 12-lead electrocardiogram showing ST-segment elevations (black arrow) and PR-depressions (blue arrow) in multiple leads, consistent with pericarditis. 


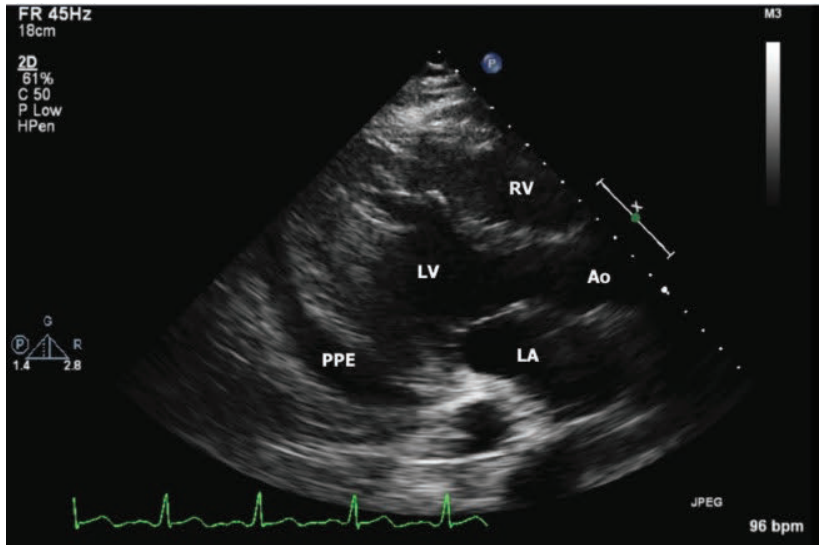

Figure 2a. A transthoracic 2-dimensional echocardiogram (parasternal long-axis view) showing a posterior pericardial effusion (PPE). LV, left ventricle; RV, right ventricle; $L A$, left atrium; Ao, aorta.

\section{DIFFERENTIAL DIAGNOSIS}

When pericarditis is complicated by a pericardial effusion, a pericardiocentesis is often recommended to relieve pressure and to rule out infectious etiologies (viral, bacterial, tuberculosis, fungal), leukemic involvement, and pericardial hemorrhage. Since our patient's pericardial effusion was only small-to-moderate in size without evidence of tamponade, and he had a high bleeding risk from thrombocytopenia, a pericardiocentesis was not performed. The absence of pericardial cultures and cytology made it challenging to definitively rule out other causes. However, he was naive to radiation therapy and the patient's infectious work up (blood and urine cultures; CT of the chest, abdomen, and pelvis; and cytomegalovirus serology) was unrevealing. Although he had acute kidney injury, his blood urea nitrogen was only mildly elevated at $33 \mathrm{mg} / \mathrm{dL}$ with a creatinine of $1.8 \mathrm{mg} / \mathrm{dl}$ (baseline unknown, but was 1.4 on admission). Rheumatological labs were not checked, but clinically, the patient did not show evidence of autoimmune disease given the absence of myalgia, arthralgia, skin or nail changes, or any evidence of an underlying vasculitis or serositis. Although it is possible that leukemic infiltration of the pericardium was the cause of the pericardial effusion, leukemic infiltration of the pericardium is very rare ${ }^{3}$. Ultimately, the temporal relationship between chemotherapy and the onset of pericarditis, as well as the absence of other potential etiologies, suggest that cytarabine was the most likely cause of this patient's presentation.

\section{OUTCOME AND FOLLOW UP}

The patient's hospitalization prior to his episode of chest pain had been complicated by acute renal failure attributed to acute tubular necrosis or cardiorenal syndrome as well

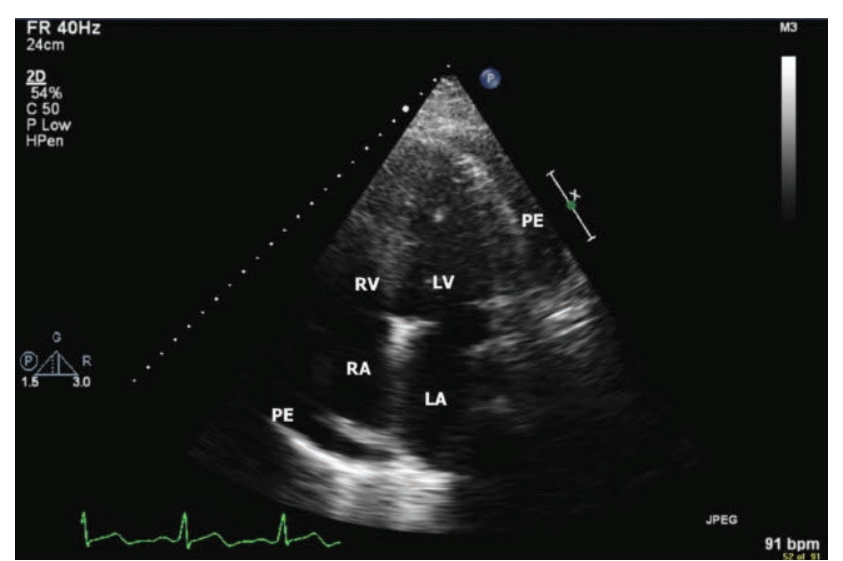

Figure 2b. A transthoracic 2-dimensional echocardiogram (apical 4-chamber view) showing a circumferential pericardial effusion (PE). LV, left ventricle; RV, right ventricle; LA, left atrium; RA, right atrium.

as neutropenic fever. Due to the acute renal failure, colchicine was not initiated. In addition, his severe thrombocytopenia limited use of aspirin and nonsteroidal anti-inflammatory drug (NSAID). Steroid therapy was discussed, but given the stability of the patient and the lack of strong evidence supporting steroid therapy, watchful management was chosen. Daily transthoracic echocardiograms revealed stable pericardial effusions. Cytarabine was never restarted. The patient eventually died after a prolonged and complicated hospitalization with renal failure requiring hemodialysis, septic thrombophlebitis with bilateral internal jugular clots, and fungemia. Of note, the fungemia developed over a week after the pericarditis; and it was felt to be from the hemodialysis catheter that was placed after the pericarditis occurred. An autopsy was not performed.

\section{DISCUSSION}

Our patient developed pericarditis and pericardial effusions after 10 doses of high dose cytarabine. The incidence of cytarabine-induced pericarditis is unknown, although there are at least nine published reports of cytarabine-induced pericarditis in the literature ${ }^{3-11}$. In addition, the manufacturer's (Upjohn) database reported six cases of pericarditis following initiation of cytarabine therapy ${ }^{3}$

The pathogenesis behind cytarabine-induced pericarditis is unclear, but some believe it is secondary to endothelial damage by cytarabine ${ }^{12,13}$. Chiche et al. found elevated levels of TNF-alpha and platelet activating factor after cytarabine therapy, suggesting that cytarabine-related pericarditis may be caused by a systemic inflammatory state amenable to steroid therapy ${ }^{14}$. Others suggest an immunologic pathogenesis of delayed hypersensitivity reaction since cytarabine-associated pericarditis often occurs after repeat administration of the drug ${ }^{3.4}$. 
The onset of cytarabine-induced pericarditis varies between 3 to 29 days following initiation of cytarabine therapy ${ }^{3-11}$. Treatment of cytarabine-induced pericarditis has not been adequately studied. NSAIDs may be helpful, but are often contraindicated in patients with hematologic malignancy due to their severe thrombocytopenia. Several authors have recommended steroid use $4,6,8$, although there have been no studies to determine the dose, route, or duration. Our patient's pericarditis remained stable without any medications. However, after reviewing the literature and understanding the likely pathogenic mechanism behind cytarabine-induced pericarditis, in future cases we would suggest treatment with high-dose steroids for cytarabine-induced pericarditis cases especially when NSAIDs are contraindicated and the patient is unstable.

Recurrence of pericarditis after cytarabine-induced pericarditis has not been formally studied, but a case report by Reykdal et al. discusses a patient who developed pericarditis on two occasions after cytarabine use (day 5 and 14$)^{3}$. In addition, Patel et al. noted that $10-30 \%$ of patients with acute pericarditis from any cause have recurrent disease ${ }^{11}$. Therefore, patients with a history of pericarditis may be at an increased risk of developing pericarditis from cytarabine, and patients who have developed cytarabine-induced pericarditis should be monitored very closely for recurrence if cytarabine is used again.

\section{KEY POINTS}

Our case illustrates a presentation of cytarabine-induced pericarditis and pericardial effusion, which is a rare but serious complication of cytarabine. Pericardial effusion can lead to potentially fatal tamponade, so a high index of suspicion is necessary for any patient receiving cytarabine, especially if they develop chest pain. Treatment with high-dose steroids should be initiated early with pericardiocentesis, if necessary. However, in patients with milder symptoms and lack of hemodynamic compromise, watchful waiting while delaying treatment can be a reasonable and safe option.

\section{REFERENCES}

1. Hamada A, Kawaguchi T, Nakano M. Clinical pharmacokinetics of cytarabine formulations. Clin Pharmacokinet. 2002;41(10):705-18.

2. Wayangankar SA, Patel BC, Parekh HD, Holter JL, Lazzara R. High-dose cytosine arabinoside-induced symptomatic bradycardia. J Cardiovasc Med (Hagerstown). 2015;16 Suppl 1:S38-41

3. Reykdal S, Sham R, Kouides P. Cytarabine-induced pericarditis: a case report and review of the literature of the cardio-pulmonary complications of cytarabine therapy. Leuk Res. 1995;19(2):141-4.

4. Gahler A, Hitz F, Hess U, Cerny T. Acute pericarditis and pleural effusion complicating cytarabine chemotherapy. Onkologie. 2003;26(4):348-50.

5. Vaickus $L$, Letendre L. Pericarditis induced by high-dose cytarabine therapy. Arch Intern Med. 1984;144(9):1868-9.

6. Hermans C, Straetmans N, Michaux JL, Ferrant A. Pericarditis induced by high-dose cytosine arabinoside chemotherapy. Ann Hematol. 1997;75(12):55-7.

7. Braverman AC, Antin JH, Plappert MT, Cook EF, Lee RT. Cyclophosphamide cardiotoxicity in bone marrow transplantation: a prospective evaluation of new dosing regimens. J Clin Oncol. 1991;9(7):1215-23.

8. Gillis K, Dundas S. Tuberculous pericarditis: a diagnostic quandary. Scott Med J. 2011;56(4):236

9. Yamada T, Tsurumi H, Hara T, Sawada M, Oyama M, Moriwaki H. ICytarabineinduced pericarditis]. Rinsho Ketsueki. 1998;39(11):1115-20

10. Woods T, Vidarsson B, Mosher D, Stein JH. Transient effusive-constrictive pericarditis due to chemotherapy. Clin Cardiol. 1999;22(4):316-8.

11. Patel A, Mainardi A, Varma M, Sara G. Cytarabine Induced Pericardial Tamponade. Blood. 2011; 118(21): 4298.

12. Boogaerts MA, Malbrain S, Verwilghen RL. The ara-c syndrome. J Haematol. 1987:66:423

13. Weiss RB. Hypersensitivity reactions. Semin Oncol. 1992;19(5):458-77.

14. Chiche D, Pico JL, Bernaudin JF, et al. Pulmonary edema and shock after high-dose aracytine-C for lymphoma; possible role of TNF-alpha and PAF. Eur Cytokine Netw. 1993;4(2):147-51.

15. Berg D. Advanced Clinical Skills and Physical Diagnosis. 2nd ed. Malden, MA:Blackwell Publishing Inc; 2004. 